\title{
Professor Plochmann Ehrenmitglied der Society of American Foresters
}

Professor Dr. Richard Plochmann, Inhaber des Lehrstuhls für Forstpolitik und Forstgeschichte und z. Z. Konrektor an der Universität München, wurde von der Society of American Foresters zum Ehrenmitglied ernannt.

\section{Deutsche Pflanzenschutztagung}

Vom 6. bis 10. Oktober 1975 findet in Oldenburg die 40. Deutsche Pflanzenschutztagung statt. Im Programm sind u. a. folgende Themenkreise vorgesehen:

Aktuelle Probleme aus dem Gebiet der Phytomedizin, Phytopharmakologie, Formulierung und Applikation von Pflanzenschutzmitteln, biotechnische Verfahren zur Schädlingsbekämpfung und anderes.

Anmeldung und Anfragen sind zu richten an die Biologische Bundesanstalt für Land- und Forstwirtschaft, D-33 Braunschweig, Messeweg 11/1?.

\section{K WF-Tagung 1975}

Die 6. Arbeitstagung des Kuratoriums für Waldarbeit und Forsttechnik fand vom 26. bis 29. Mai 1975 in Braunschweig statt. Das Thema lautete: Durchforstung.

\section{IIl. BUCHBESPRECHUNGEN}

Shifting cultivation in Latin America. By R. F. WAtTers. FAO Forestry Development Paper No. 17, Rom 1971. 305 S.

Der Brandrodungs-Wanderfeldbau - englisch: shifting cultivation - ist eine Bodennutzung, die vor allem in tropischen und subtropischen Regionen weit verbreitet ist. Schon lange vor dem industriellen Zeitalter war er eine wichtige Form der Produktion von Nahrungsmitteln für die dort ansässigen Volksstämme. Eine Waldparzelle, deren Größe von der benötigten Nahrungsmittelmenge abhängt, wird gesäubert (keine Stockrodung) und abgebrannt. Nach meist primitiver Bodenbearbeitung mit der Hadke kann 1-3 Jahre lang gesät und geernter werden. Starke Verunkrautung und schneller Verlust der Bodenfruchtbarkeit machen es dann notwendig, eine neue Waidfläche anzugreifen und die alte sich selbst zu überlassen, was fast immer rasch zu einer Wiederbestodkung mit Wald - Sekundärwald - führt. Hat sich der Bodenzustand unter dieser Waldbrache wieder regeneriert, so kann an eine ncue landwirtschaftliche Nurzung gedacht werden. Dieses Bodennutzungssystem war so lange unbedenklich, wie die Bevölkerungszahlen nicht sehr hoch lagen und die "primiriven" Bewohner solcher Waldgebiete aus Erfahrung und Einfühlungsvermögen in ökologische Zusammenhänge das Verhältnis zwischen Kultur- und Bracheperiode so wählten, daß die Produktionskraft des Bodens nicht beeinträchtigt wurde. Rasches Bevölkerungswachstum und damit der $Z_{w a n g}$, mehr Wald in diese Form der landwirtschaftlicien Nutzung einzubeziehen und die Brachezeiten zu verkürzen, brachten jedoch die Anpassung an die natürlichen Möglichkeiten durcheinander. Hinzu k:am besonders in Lareinamerika das Auftauchen nicht eingeborener Siedler, die entlang aller Kommunikationslinien eine besonders wenig ökologisch fundierte Form der shifting cultivation begannen. Heute steilt der Brandrodungs-Wanderfeldbau eines der schwierigsten 